\title{
Congenital Portosystemic Shunt as a Rare Cause of Neonatal Cholestasis: Case Report
}

\author{
(1) Cemil Oktay¹, (1) Sibel Yavuz², (1) Mehmet Emin Parlak³, (1) Gökhan Özel3 \\ ${ }^{1}$ Adıyaman University Training and Research Hospital, Clinic of Radiology, Adıyaman, Turkey \\ 2Adıyaman University Training and Research Hospital, Clinic of Pediatric Gastroenterology, Adıyaman, Turkey \\ ${ }^{3}$ Adıyaman University Training and Research Hospital, Clinic of Pediatrics, Adıyaman, Turkey
}

\section{ABSTRACT}

Cholestatic jaundice is a complex diagnostic problem with a wide spectrum of possible differential diagnosis in early infancy. We present a case of congenital intrahepatic portosystemic venous shunt as a rare cause of neonatal cholestasis with current treatment recommendations.

Keywords: Portosystemic shunt, neonatal cholestasis, ultrasonography

\section{Introduction}

Congenital portosystemic shunts (CPSS) are rare developmental vascular abnormalities with an incidence of about one in 30,000 children (1). These shunts cause a diversion of portal venous flow to the systemic venous system, and thus, splanchnic portal venous blood bypasses the liver parenchyma and various biochemical metabolites (galactose, ammonia, and bile acids etc.) accumulate in the systemic circulation without being metabolized (1). Depending on the accumulated amount of these metabolites, clinical presentation can be quite variable, especially in children. CPSS can either be seen in a symptomatic infant or detected incidentally in a child that undergoes ultrasonography (US) for other reasons. We present a case who underwent abdominal US because of neonatal cholestasis and was diagnosed with CPSS.

\section{Case Report}

An 18-day-old male patient presented with a complaint of jaundice which started when he was 3 days old. It was discovered that the patient was born via spontaneous vaginal delivery with a weight of 2,500 gr at the $36^{\text {th }}+6$ week of gestation from a 27-year-old mother with normal pregnancy period. There was no consanguinity between the parents and there was no history of sibling death. In physical examination, his general condition was good and active, and the patient's body weight was found to be $3,000 \mathrm{gr}\left(3-10^{\text {th }}\right.$ percentile), and his height was $50 \mathrm{~cm}\left(10-25^{\text {th }}\right.$ percentile). Sclera and skin were icteric. No evidence of congestive heart failure or hepatosplenomegaly was found.

Laboratory findings were as follows; hemoglobin 12 $\mathrm{g} / \mathrm{dL}$ mean corpuscular volume $88 \mathrm{fL}$, leukocyte count $9,100 / \mathrm{mm}^{3}$, platelet count $185,000 / \mathrm{mm}^{3}$, serum aspartate transaminase $46 \mathrm{U} / \mathrm{L}$, alanine transaminase $8 \mathrm{U} / \mathrm{L}$, gammaglutamyltransferase $219 \mathrm{U} / \mathrm{L}$, alkaline phosphatase $126 \mathrm{U} / \mathrm{L}$, total protein $6.1 \mathrm{~g} / \mathrm{dL}$, albumin $3.8 \mathrm{~g} / \mathrm{dL}$, ammonia $60 \mu \mathrm{g} / \mathrm{dL}$, total/direct bilirubin $7.3 / 4.8 \mathrm{mg} / \mathrm{dL}$, phosphorus $3.8 \mathrm{mg} / \mathrm{dL} . \mathrm{In}$ addition, kidney function tests, serum electrolytes and blood glucose were normal. Arterial blood gas, thyroid function

\section{Address for Correspondence}

Cemil Oktay, Adıyaman University Training and Research Hospital, Clinic of Radiology, Adıyaman, Turkey Phone: +90 5065118206 E-mail: cemiloktay@gmail.com ORCID: orcid.org/0000-0002-1595-8014 Received: 18.11.2020 Accepted: 01.01.2021

${ }^{\circ}$ Copyright 2021 by Ege University Faculty of Medicine, Department of Pediatrics and Ege Children's Foundation The Journal of Pediatric Research, published by Galenos Publishing House. 
test, alpha-1 antitrypsin level, ferritin, complete urinalysis, prothrombin time, activated partial thromboplastin time were normal, and serological examinations for hepatitis A, B and C viruses, cytomegalovirus, Epstein-Barr virus, toxoplasma, rubella and human immunodeficiency virus were negative. In screening for hereditary metabolic disease, the reducing substance in the urine was negative, and urineblood amino acids and organic acid analysis in the urine by tandem mass spectrophotometer were normal. Blood and urine culture were normal. Echocardiographic evaluation was normal. Eye examination revealed no signs of metabolic disease or cataract. Abdominal ultrasonography was performed for neonatal cholestasis.

In ultrasonography, normal liver contours and parenchymal echo pattern, and parenchymal millimetric rough calcifications in the right subdiaphragmatic area were observed. The gallbladder was normal and no cord sign was noted. Common bile duct and intrahepatic bile ducts were normal. Doppler ultrasonography examination showed that the main portal vein and hepatic artery were normal in diameter and flow pattern. However, the left portal vein diameter was increased and the right portal vein was significantly hypoplastic. Also, there was an aberrant venous connection $(3 \times 1.5 \mathrm{~mm})$ between the inferior vena cava and portal vein at the bifurcation level (Figure 1). Hepatic veins showed normal phasic flow and diameter but in the inferior vena cava, there was increased turbulent flow and diameter at the shunt level. There was no evidence of portal hypertension. Liver computed tomography angiography was performed to confirm the findings described on ultrasound and to evaluate other vascular structures, and a single isolated shunt was detected on tomography (Figure 2).

Vitamins $A, D, E, K$ and ursodeoxycholic acid were started as treatment. The patient, who did not develop complications such as portal hypertension, heart failure or hepatopulmonary syndrome during the follow-up, was discharged after 1 week of hospitalization with good activity, nutrition and weight gain. The family was informed about possible complications and called for regular follow-ups. In the check-up ultrasound examination that was performed in the $3^{\text {rd }}$ month, the shunt was completely closed, and band-like linear hyperechogenicity was observed in this area (Figure 1). In addition, the hypoplastic right portal vein diameter was increased compared to the previous examination and the left portal vein diameter had returned to normal (Figure 1). Over time, sclera and skin returned to normal colors and the bilirubin values returned to normal in the sixth month of follow-up. During the whole follow-up,

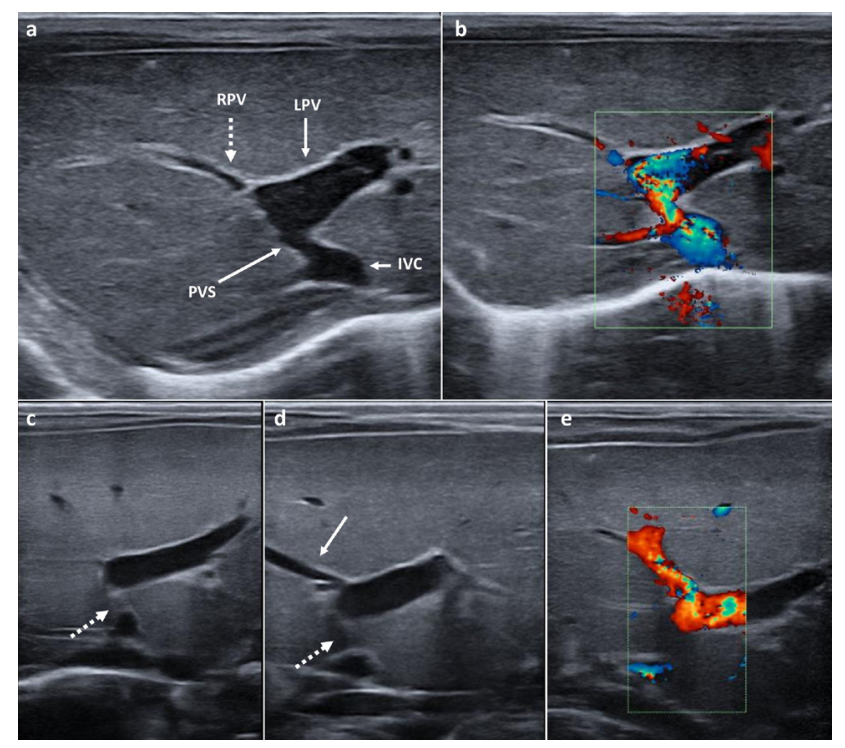

Figure 1. Ultrasound images at the time of diagnosis (a-b) and at third month check-up (c-e). Figure 1a shows the hypoplastic right portal vein (RPV) and the left portal vein (LPV) with increased diameter. Also, porto venous shunt (PVS) is observed between the inferior vena cava (IVC) and the portal vein at the bifurcation level (type 1 CPSS). Figure $1 \mathrm{~b}$ shows increased turbulent flow and related aliasing artifacts at the shunt level in Doppler ultrasonography. Figure $1 \mathrm{c}$ and $1 \mathrm{~d}$ show the closed shunt (dotted arrow) and linear echogenicities in this area. Gray scale (Figure 1d) and Doppler (Figure 1e) ultrasound images of the right portal vein (straight arrow) shows the increased diameter at the $3^{\text {rd }}$ month checkup, compared to Figure 1a
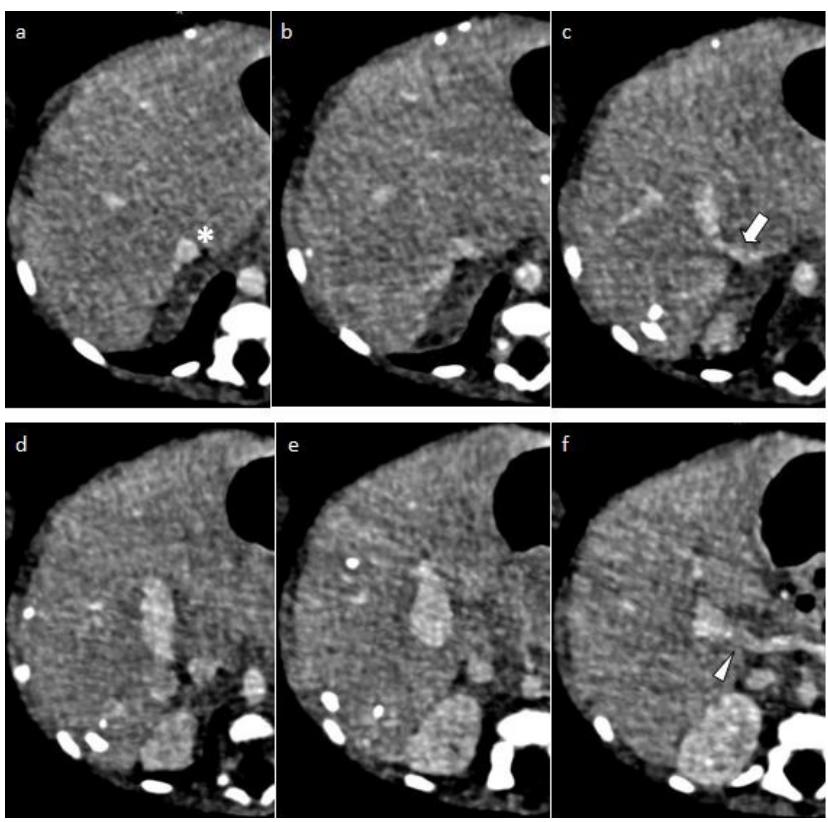

Figure 2. In the axial plane serial images of liver computed tomography angiography; the inferior vena cava (asterisk) is seen in Figure $2 a$ (asterisk) and Figure 2b. Figure $2 \mathrm{c}$ shows the shunt extending from the portal bifurcation to the inferior vena cava (white arrow). While enlarged portal bifurcation is observed in Figure $2 \mathrm{~d}$ and $2 \mathrm{e}$, the main portal vein (arrowhead) observed in normal calibration is seen in Figure $2 f$ 
the patient had a lively appearance with normal sleep habits and diet for his age.

The authors declare that informed consent was obtained from the parents for the publication of this case report.

\section{Discussion}

CPSS are rare, developmental anatomical abnormalities, resulting in a diversion of portal venous flow to the systemic venous system. CPSS are divided into extrahepatic (abernethy malformation) and intrahepatic types according to their anatomical features (1). Congenital intrahepatic shunts were subdivided into 4 morphological types by Park et al. (2) as follows;

Type 1: a single large shunt connecting the right portal vein to the inferior vena cava

Type 2: a localized peripheral shunt in one hepatic segment where one or more communications are found

Type 3: a communication between the peripheral portal vein and the hepatic veins through a portal vein "aneurysm"

Type 4: multiple and diffuse portosystemic shunts in several segments

CPSS can cause a wide range of clinical symptoms, ranging from asymptomatic patients to those with severe symptoms and complications. Hepatic encephalopathy, pulmonary hypertension, and hepatopulmonary syndrome are the most outstanding symptoms and portosystemic shunt cases can lead to a large extent of metabolic irregularities, while damage to other organs can be seen in a very few cases (3). In the perinatal period, neonatal cholestasis, hyperammonemia, hypoglycemia and hypergalactosemia can occur and should be differentiated from other congenital anomalies such as biliary atresia and accompanying metabolic diseases $(4,5)$.

Due to the wide variability in clinical manifestation, imaging plays a crucial role in the recognition of shunt and accompanied malformations. Doppler ultrasonography is the key imaging modality to show the presence of the shunt and its type. In addition, Doppler ultrasonography can be used to confirm the direction of shunt flow, to calculate the shunt ratio (by dividing the total blood flow volume at the shunt by the total portal vein flow) and for the follow-up of CPSS (6).

Abdominal cross-sectional imaging can provide additional information about the shunt anatomy and characterize any potential focal liver lesion. Although MR angiography can decrease the ionizing radiation exposure especially in pediatric patients, computed tomography angiography is considered the first line screening method (7). This is due to the fact that this method can show even small portosystemic shunt branches properly.

Currently, there is no standard therapeutic approach available for portosystemic shunts which has been adopted for large studies. Treatment options are shaped according to the type of shunt and its clinical course. For those patients with asymptomatic intrahepatic shunts, as is frequently the case in children, follow-up without treatment is recommended. Clinical manifestations are frequently seen in adulthood or in those patients with a shunt ratio greater than 60\%; and treatment is recommended at the onset of clinical manifestations $(8,9)$. To date, there is no guide recommending early treatment to prevent undeveloped complications. In those patients with mild clinical signs, intrahepatic small shunts may regress within one year and close spontaneously with a resolution of symptoms (7). Spontaneous closure of intrahepatic shunts often occurs in children with newborn cholestasis, in girls and in those patients with type 4 shunts (10). In contrast to these, extrahepatic shunts and patent ductus venosus are less likely to close (10). It is recommended that all shunts which persist after 1 year of age should be closed regardless of whether complications develop or not (7). The main goal of the treatment is to close the shunt without causing secondary portal hypertension, and shunt occlusion options vary from the least invasive percutaneous endovascular procedures to surgical correction with liver transplantation as a last resort (6).

Intrahepatic portosystemic shunts are unusual vascular anomalies which might be detected as a cause of neonatal cholestasis. This pathology, which can only be diagnosed radiologically, should be on the differential diagnosis list in each examination that is performed for cholestasis. Familiarity with Doppler ultrasonography features of intrahepatic portosystemic shunts could help to diagnose affected patients and to choose the best therapeutic approach

\section{Ethics}

Informed Consent: The authors declare that informed consent was obtained from the parents for publication of the case.

Peer-reviewed: Externally peer-reviewed.

\section{Authorship Contributions}

Data Collection or Processing: M.E.P., G.Ö., Analysis or Interpretation: M.E.P., G.Ö., S.Y., Literature Search: C.O., Writing: C.O. 
Conflict of Interest: No conflict of interest was declared by the authors.

Financial Disclosure: The authors declared that this study received no financial support.

\section{References}

1. Bernard O, Franchi-Abella S, Branchereau S, Pariente D, Gauthier $F$, Jacquemin E. Congenital portosystemic shunts in children: recognition, evaluation, and management. Semin Liver Dis 2012; 32:273-87.

2. Park JH, Cha SH, Han JK, Han MC. Intrahepatic portosystemic venous shunt. AJR Am J Roentgenol 1990; 155:527-8.

3. Timpanaro T, Passanisi S, Sauna A, et al. Congenital portosystemic shunt: our experience. Case Rep Pediatr 2015; 2015:691618.

4. Nagasaka H, Miida T, Yorifuji T, et al. Metabolic improvements in intrahepatic porto-systemic venous shunt presenting various metabolic abnormalities by 4-phenylacetate. Clin Chim Acta 2013; 419:52-6.
5. Yamagami T, Nakamura T, Tokiwa K, et al. Intrahepatic portosystemic venous shunt associated with biliary atresia: case report. Pediatr Radiol 2000; 30:489-91.

6. Hu GH, Shen LG, Yang J, et al. Insight into congenital absence of the portal vein: is it rare? World / Gastroenterol 2008; 14:596979 .

7. Papamichail M, Pizanias M, Heaton N. Congenital portosystemic venous shunt. Eur / Pediatr 2018; 177:285-94.

8. Gallego C, Miralles M, Marín C, et al. Congenital hepatic shunts. Radiographics 2004; 24:755-72.

9. Akahoshi T, Nishizaki T, Wakasugi K, et al. Portal-systemic encephalopathy due to a congenital extrahepatic portosystemic shunt: threecases and literature review. Hepatogastroenterology 2000; 47:1113-6.

10. Paganelli M, Lipsich JE, Sciveres M, et al. Predisposing factors for spontaneous closure of congenital portosystemic shunts. I Pediatr 2015; 167:931-5. 